\title{
Results and molecular correlates from a pilot study of neoadjuvant induction FOLFIRINOX followed by chemoradiation and surgery for gastroesophageal adenocarcinomas
}

Jennifer Wo ( $\square$ JWO@mgh.harvard.edu )

Massachusetts General Hospital

Jeffrey Clark

Massachusetts General Hospital

Christine Eyler

Duke University

Mari Mino-Kenudson

Massachusetts General Hospital https://orcid.org/0000-0002-9092-2265

Samuel Klempner

Massachusetts General Hospital

Jill Allen

Massachusetts General Hospital

Florence Keane

Massachusetts General Hospital

Aparna Parikh

Massachusetts General Hospital https://orcid.org/0000-0002-5245-7841

Eric Roeland

Massachusetts General Hospital

Lorraine Drapek

Massachusetts General Hospital

David Ryan

Massachesetts General Hospital

Ryan Corcoran

Massachusetts General Hospital https://orcid.org/0000-0001-8173-5778

Emily van Seventer

Massachusetts General Hospital

Isobel Fetter

University of California, Santa Cruz

Heather Shahzade

Massachusetts General Hospital 


\section{Melin Khandekar}

Massachusetts General Hospital

\section{Michael Lanuti}

Massachusetts General Hospital

\section{Christopher Morse}

Massachusetts General Hospital

\section{Rebecca Heist}

Massachusetts General Hospital

Christine Ulysse

Massachusetts General Hospital

\section{Benjamin Christopher}

Massachusetts General Hospital

\section{Christian Baglini}

Massachusetts General Hospital

\section{Beow Yeap}

Massachusetts General Hospital

John Mullen

Massachusetts General Hospital

Theodore Hong

Massachusetts General Hospital

\section{Article}

Keywords: FOLFIRINOX, CRT, esophageal cancer, gastrointestinal cancer, tumor

Posted Date: November 9th, 2020

DOI: https://doi.org/10.21203/rs.3.rs-92371/v1

License: (c) (1) This work is licensed under a Creative Commons Attribution 4.0 International License. Read Full License

Version of Record: A version of this preprint was published at Clinical Cancer Research on July 30th, 2021. See the published version at https://doi.org/10.1158/1078-0432.CCR-21-0331. 


\section{Abstract}

We performed a NCl-sponsored, prospective study of neoadjuvant FOLFIRINOX followed by chemoradiation (CRT) with carboplatin/paclitaxel followed by surgery in patients with locally advanced gastric or gastroesophageal (GEA) cancer. The primary objective was to determine completion rate of neoadjuvant FOLFIRINOX x 8 followed by CRT. Secondary endpoints were toxicity and pathologic complete response $(\mathrm{pCR})$ rate. Exploratory analysis was performed of ctDNA to treatment response. From Oct 2017 to June 2018, 25 patients were enrolled. All patients started FOLFIRINOX, 92\% completed all 8 planned cycles, and $88 \%$ completed CRT. Twenty $(80 \%)$ patients underwent surgical resection, and 7 had a pCR (35\% in resected cohort, $28 \%$ ITT ). Tumor-specific mutations were identified in 21 (84\%) patients, of whom 4 and 17 patients had undetectable and detectable ctDNA at baseline, respectively. Presence of detectable post-CRT ctDNA $(p=0.004)$ and/or postoperative ctDNA $(p=0.045)$ were associated with disease recurrence. Here we show neoadjuvant FOLFIRINOX followed by CRT for locally advanced GEA is feasible and yields a high rate of pCR. ctDNA appears to be a promising predictor of postoperative recurrence.

\section{Introduction}

Despite recent advancements, gastric and gastroesophageal junction adenocarcinoma (GEA) remains a lethal disease ${ }^{1,2}$. While surgical resection offers the potential for cure in locoregional disease, the majority of patients experience disease recurrence within 5 years $^{3}$. To that end, numerous treatment paradigms and regimens have been explored to improve upon the outcomes of surgery alone ${ }^{3-9}$. For gastric cancer, postoperative chemoradiation, perioperative chemotherapy, and postoperative chemotherapy are accepted approaches ${ }^{3-8}$. For locally advanced GEJ cancer, preoperative chemoradiation and perioperative chemotherapy have been established as standards of care ${ }^{4,9}$. However, despite these recent advancements, the risk of distant recurrence remains high, at over $50 \%$ by five years ${ }^{3,5}$. Strategies to optimize the integration and sequencing of multimodality therapy, coupled to biomarkers of response, remain a significant unmet need ${ }^{10-12}$.

Outside of GEA, FOLFIRINOX has emerged as a standard of care for pancreatic cancer in both the resectable and metastatic setting ${ }^{13,14}$. Additionally, there is a growing body of literature evaluating the efficacy of this regimen in gastroesophageal and gastric cancers ${ }^{15-17}$. A pooled analysis of two ongoing Phase II trials of FOLFIRINOX in the first line metastatic setting for GE junction cancers demonstrated an overall response rate (ORR) of $62.5 \%{ }^{15}$. In a recently published phase II trial of advanced GEA, among ERBB2-negative patients treated with FOLFIRINOX alone, the ORR was $61 \%$, with a median overall survival of 15.5 months. Among ERBB2-positive patients treated with FOLFIRINOX and trastuzumab, the ORR was $85 \%$, with a median OS 19.6 months ${ }^{16}$. Additionally, a multi-institutional phase II trial of 36 patients with locally advanced GEA evaluated pharmacogenomically dosed perioperative FOLFIRINOX and reported R0 resection rate of $92 \%$ and a pathologic complete response (pCR) rate of $23 \%{ }^{17}$. Based on these promising results, we sought to perform a single-arm pilot study of total neoadjuvant therapy 
with FOLFIRINOX, followed by consolidative chemoradiation with concurrent carboplatin/paclitaxel, followed by surgery, in patients with GEA planned for curative intent therapy.

\section{Methods}

\section{Patient Selection:}

Patients with previously untreated gastric or gastroesophageal junction adenocarcinoma were enrolled in this National Cancer Institute-sponsored single-arm pilot clinical trial from October 2017 to June 2018 (NCT03279237). This study was approved by the XXXX/XXXX Institutional Review Board. All patients provided written informed consent. Prior to study enrollment, all patients underwent the following evaluation: 1) clinical staging, including EGD +/- EUS, computed tomographic (CT) scan of the chest and either a CT scan of the abdomen/pelvis or a magnetic resonance imaging (MRI) scan of the abdomen with intravenous (IV) contrast within 42 days of enrollment, 2) physical examination within 14 days of enrollment, and 3) baseline laboratory studies, including urine HCG for women of childbearing potential. Staging laparoscopy was not required prior to study treatment and was at the discretion of the treating investigator.

Study eligibility criteria were as follows: histologically or cytologically confirmed T3/4 or lymph node positive disease (defined as $>1 \mathrm{~cm}$ in size and/or FDG-avid), centralized pathologic confirmation; age $\geq 18$ years; ECOG PS $\leq 1$; life expectancy $>3$ months; and adequate organ and marrow function as defined as absolute neutrophil count $\geq 1,500$ cells $/ \mathrm{mm}^{3}$; platelets $\geq 75,000$ cells $/ \mathrm{mm}^{3}$; AST(SGOT) and ALT (SGPT) $\leq 2.5 \times$ upper limit of normal, and creatinine $\leq 1.5 \mathrm{mg} / \mathrm{dL}$, or creatinine clearance $\geq 30$ $\mathrm{mL} / \mathrm{min} / 1.73 \mathrm{~m}^{2}$ for participants with creatinine levels above institutional normal; and the ability to understand and the willingness to sign a written informed consent document. The full protocol is provided in supplemental data (Supplemental Appendix 1).

Exclusion criteria included: evidence of metastatic disease within six weeks of study entry, prior treatment for the participant's gastric or GE junction cancer; treatment of other invasive carcinomas within the last five years with greater than $5 \%$ risk of recurrence at time of eligibility screening; receipt of any other investigational agents within 4 weeks preceding the start of study treatment; serious concomitant systemic disorders; pregnancy; major surgery (excluding laparoscopy) within 4 weeks of start of study treatment; prior systemic fluoropyrimidine therapy or known hypersensitivity to 5-fluorouracil or known dihydropyrimidine dehydrogenase (DPD) deficiency; and a history of allergic reaction(s) attributed to compounds of similar chemical or biologic composition to 5 -fluorouracil, irinotecan, or oxaliplatin. Distant nodal disease was allowed if it was deemed to be covered within a radiation treatment field and curative therapy was planned. 
This is a single-arm pilot study of the neoadjuvant administration of the FOLFIRINOX regimen and preoperative radiation therapy followed by surgery in patients with locally advanced ( $\mathrm{T} 3 / 4$ or $\mathrm{N}+$ ) gastric or GEA. The primary study objective was to determine the completion rate of neoadjuvant FOLFIRINOX followed by consolidative chemoradiation with concurrent carboplatin/paclitaxel delivered in the preoperative setting. Secondary objectives included: 1) acute toxicity; 2) pCR rate; 3) progression-free survival (PFS); 4) overall survival (OS). Pre-planned correlative studies to explore the relationship between ctDNA and clinical outcomes were performed. Exploratory analyses were performed to evaluate tumor microenvironment and molecular profiling.

\section{FOLFIRINOX}

FOLFIRINOX was planned for 8 cycles on a 14-day cycle. 5-Fluorouracil was administered as 400 $\mathrm{mg} / \mathrm{m}^{2}$ bolus on day 1 , then as a $2400 \mathrm{mg} / \mathrm{m}^{2}$ continuous infusion for 46 hours. Leucovorin calcium, $400 \mathrm{mg} / \mathrm{m}^{2}$; oxaliplatin $85 \mathrm{mg} / \mathrm{m}^{2}$, and irinotecan hydrochloride $180 \mathrm{mg} / \mathrm{m}^{2}$, were administered on day 1 , as previously described ${ }^{14}$. Pegfilgrastim, $6 \mathrm{mg}$, was administered on day 4 . Dose adjustments for toxicity were predefined in the protocol (Supplemental Appendix 2). Patients were restaged with CT scans of the chest, abdomen, and pelvis after 4 and 8 cycles of FOLFIRINOX.

Chemoradiation

Chemoradiation treatments were scheduled to begin within 4 weeks after completion of FOLFIRINOX. Carboplatin and paclitaxel were administered concurrently with the radiation therapy. Carboplatin (area under the curve $[A U C]=2$ ) was administered weekly over 1 hour. Paclitaxel $50 \mathrm{mg} / \mathrm{m}^{2}$ was administered as an IV infusion over 30-60 minutes weekly as previously described ${ }^{9}$.

All radiation treatments were administered at the XXXXX. All patients underwent 4D-CT simulation with oral and IV contrast in a supine position. For photons, all patients received IMRT or VMAT. Daily cone beam CT was performed pre-treatment for set up verification. Tumor volume was defined on the basis of $\mathrm{CT}, \mathrm{PET} / \mathrm{CT}$ and MRI. The Gross Tumor Volume (GTV) was defined as the gross primary tumor and any lymph nodes $\geq 1 \mathrm{~cm}$. For participants with diffuse type gastric cancer, where the GTV could not be visualized, the entire stomach was identified as GTV. When the GTV was clearly identified, the clinical target volume (CTV) included a longitudinal mucosal margin of 3.5-4 cm proximally and distally to the GTV. A $5 \mathrm{~mm}$ CTV expansion was generated based on all grossly enlarged lymph nodes. Elective lymph node coverage included celiac and gastrohepatic lymph nodes for all patients. For GEJ and cardia lesions, coverage of paraesophageal lymph nodes within $4 \mathrm{~cm}$ of the tumor was recommended; for distal gastric lesions, porta hepatis lymph node coverage was recommended. Coverage of splenic lymph nodes (for GE junction/cardia lesions) and peripancreatic lymph nodes (for distal gastric lesions) were at the discretion of the treating physician. The Planning Target Volume (PTV) was customized based on the 
4D CT scan; however, generally, a $5 \mathrm{~mm}$ expansion was used, except for $7 \mathrm{~mm}$ superiorly/inferiorly. The prescribed dose to the PTV for gastric tumors was $4500 \mathrm{cGy}$ delivered in $180 \mathrm{cGy} /$ day over 25 fractions. For gastroesophageal junction tumors, a 540 cGy boost was included to the GTV $+1 \mathrm{~cm}$. Normal dose constraints were predefined in the protocol (Supplemental Appendix 3).

\section{Surgery}

After completion of chemoradiation, restaging CT scan was evaluated by the multidisciplinary team. Surgical resection was performed approximately 4-6 weeks after completion of chemoradiation. Surgical procedure and extent of LN dissection was performed at the discretion of the treating surgeon. For gastric cancers, an extended (D1+ or D2) lymph node dissection was performed.

Follow-Up

After treatment completion, study participants had a follow-up baseline CT scan performed 3-8 weeks postoperatively. Subsequently, follow-up visits were scheduled with laboratory evaluations every 3 months and with CT scans every 6 months for the first 2 years, visits with blood work every 3 months and annual CT scans for year 3, and visits with blood work at least every 6 months and annual CT scans up to year 5 .

\section{Pathologic Evaluation}

Pathologic findings were scored per standard institutional practices, including margin status (proximal, distal, radial) and nodal status (total assessed, total positive). Tumor regression grade (TRG) ${ }^{18}$ was recorded for all patients undergoing surgery, and pathologic complete response was defined as TRG 0 .

\section{Correlative Studies:}

\section{Circulating tumor DNA}

Tumor biopsies from the time of diagnosis as part of routine care were subjected to an internal nextgeneration sequencing platform evaluating 104 known cancer genes. Blood was drawn at pre-specified study time points (baseline, cycle 1 day 8 , cycle 5 day 1, pre-chemoradiation, preoperatively, every 3 months postoperatively, and at progression) at time of draws for routine clinical care. ctDNA was extracted from plasma using QIAGEN-based protocols and amplified by digital droplet PCR (ddPCR) 
using primers for tumor-specific point mutations. One or more driver mutations that matched the patient's tumor sequencing were followed longitudinally.

\section{Tumor Microenvironment}

Immunohistochemical staining to evaluate tumor microenvironment was performed with the remaining tissue available from the diagnostic biopsy after ctDNA analysis. Surgical resection samples were also obtained for comparison. Five-micron sections were cut from the selected paraffin blocks and stained by immunohistochemistry (IHC). CD8+ TIL were scored based on \% of tumor cells with CD8+ T cells on them as follows ${ }^{19}$ : 0 -rare; $1<5 \% ; 2-\geq 5 \%$ but $<25 \%$; $3->25 \%$. In the pretreatment specimens, 3 high power fields (HPFs) were reviewed, and the average was reported, except for PD-L1 which was reported as combined positive score (CPS). In the post-treatment cases, due to heterogeneity in immune cell infiltration appreciated, semiquantitative scoring was performed (Supplemental Appendix 4). The specific antibodies and conditions used for each immune marker were: (1) CD8 - clone 4B11, Leica Biosystem; (2) PD-1 - clone EH33, Cell Signaling Technology; (3) PD-L1 - clone E1L3N, Cell Signaling Technology; (4) CD3 - clone LN10, Leica Biosystem; (5) FOXP3 - clone 236A/7, Biocare. Due to the limited sample size, statistical analysis was not performed for paired comparison.

Tumor Molecular Analysis by SNaPshot analysis

Molecular profiling of hotspot SNP, indel and copy number variants was performed using SNaPshot analysis, an Anchored Multiplex PCR strategy previously described ${ }^{20}$. Briefly, after pathologic review to determine sample tumor enrichment, genomic DNA was isolated from FFPE- embedded tumor specimens. Genomic DNA was sheared, end-repaired, A-tailed and then modified by ligation of halffunctional Illumina adapters. Two hemi-nested PCR reactions targeting genomic regions of interest (i.e., SNP/mutation, indel or copy number hotspots, Supplemental Appendix 5) were performed, and sequenced using Illumina Nextseq 2x150bp paired-end sequencing. Genome alignment to the hg19 reference sequence was performed using Novoalign (Novocraft Technologies; Selangor, Malaysia) and various methods for variant calls were employed, including use of MuTect ${ }^{21}$, LoFreq ${ }^{22}$, GATK $^{23-25}$ and a laboratory-developed hotspot and copy number caller. This test is validated to detect variants with at least $5 \%$ allelic frequency, in regions with sufficient NGS read coverage.

Analysis of the Cancer Genome Atlas (TCGA) Data

Data from the TCGA $^{26}$ were analyzed using cBioportal ${ }^{27}$ and Xenabrowser.net ${ }^{28}$ to assess co-mutation frequency and alteration subtype specificity. 
Statistical Analysis:

The primary endpoint was neoadjuvant therapy completion, defined as the portion of patients receiving all planned induction FOLFIRINOX followed by chemoradiation. Sample size was planned for 25 patients, and if at least 18 of the 25 patients were able to complete the specified treatment plan, then the primary endpoint was met. The decision rule was associated with $89 \%$ power for declaring success if FOLFIRINOX in combination with chemoradiation were associated with an underlying completion rate of $80 \%$. In contrast, the probability of a type I error was only $15 \%$ if $60 \%$ of patients were able to complete the intended treatment plan. Toxicity was defined per CTCAE v4.03. PFS and OS were measured from the date of treatment start and estimated by the Kaplan-Meier method. OS time was censored at the date of last follow-up for patients still alive. PFS time was defined until detection of locoregional recurrence, distant metastases, or death without documented progression, whichever date was earliest, or censored at the date of last follow-up. The distant metastasis (DM) rate was estimated as the cumulative incidence with progression or death before chemoradiation as a competing risk. Fisher's exact test and Gray's test were used, respectively, to analyze PCR and DM rates by ctDNA detection status, with the p-values based on a two-sided hypothesis. As the biomarker analysis was exploratory, the level of type 1 error was not pre-specified. Statistical analysis was performed using SAS version 9.4 (SAS Institute) and R version 3.3.1 (R Foundation).

\section{Results}

Between October 2017 and June 2018, a total of 25 eligible patients were enrolled. Table 1 describes baseline patient and tumor characteristics. The median age was 60 (range: $30-76$ ), and $68 \%$ were male. The primary site was $60 \%$ GE junction and $40 \%$ stomach. The clinical stage of disease was T3N0 (8\%), T4N0 (12\%), T1-4N+ (80\%). At time of presentation, $28 \%$ of patients had nodal M1 disease.

\section{Treatment Completion}

Of 25 patients who started treatment, 2 patients developed metastatic progression on FOLFIRINOX. Of the remaining 23 patients who completed FOLFIRINOX, 23/23 (100\%) proceeded to chemoradiation. One patient died during chemoradiation of a cardiac pulseless electrical activity (PEA) arrest, which was deemed unrelated to treatment. Of the remaining 22 patients (88\%) who completed chemoradiation, all proceeded to surgical exploration. Thus, 22/25 (88\%) enrolled patients completed the induction FOLFIRINOX and chemoradiotherapy. During surgery, 2 of the 22 patients were found with intraoperative metastases, and therefore, a total of 20 patients $(80 \%)$ underwent potentially curative surgical resection (Figure 1). Among the 4 patients who developed metastatic progression over the course of treatment, none had nodal M1 disease at presentation.

Treatment Outcomes 
Of the 20 patients that went to resection, the median residual tumor size was $0.25 \mathrm{~cm}$ (range, 0.0-7.7 cm). Six (30\%) patients had positive lymph nodes, and all patients had a R0 resection. Surgical outcomes are summarized in Table 2. Seven patients achieved a pCR, corresponding to $35 \%$ of the resected patients and $28 \%$ of the entire cohort by intention to treat (ITT).

The median follow-up of the 18 surviving patients was 23.1 months (range, 19.2-26.9 months). Among the 20 patients who underwent resection, 14 are still alive without evidence of relapse. In the entire cohort, the 1-year DM rate, PFS and OS were $27 \%, 64 \%$, and $76 \%$, respectively; the 2-year DM, PFS and OS rate were $37 \%, 55 \%$, and $72 \%$, respectively (Figure 2). Excluding the 7 patients with nodal M1 disease, the 1-year and 2-year DM rate, PFS and OS were $25 \%$ and $39 \%, 67 \%$ and $54 \%$, and $78 \%$ and $72 \%$, respectively. Among the 8 patients with progression following chemoradiation, the site of first failure included the liver $(n=3)$, peritoneum $(n=3)$, lung $(n=1)$, and brain $(n=2)$.

\section{Acute Toxicity}

Table 3 summarizes the acute toxicities of this treatment regimen. The overall rates of acute grade 3 and 4 toxicity were $16 \%$ and $80 \%$, respectively. However, in all but 2 patients (8\%), grade 4 toxicity was secondary to subclinical lymphopenia during chemoradiation. To that end, the rate of non-hematologic grade $3+$ and grade $4+$ worst toxicity was $48 \%$ and $0 \%$, respectively. One patient died during the course of chemoradiation at $46.8 \mathrm{~Gy}$ from cardiac arrest (PEA arrest), which was clinically most consistent with pulmonary embolus and felt to be unrelated to treatment.

\section{ctDNA as a Prognostic Biomarker}

All patients had tissue available for tumor- specific mutational analysis. Across all patients, tumorspecific mutations were identified in 21 (84\%) patients by ctDNA ddPCR. Of the 21 patients with an evaluable mutation for ddPCR, 4 patients had undetectable ctDNA at baseline, and 17 had detectable ctDNA pre-treatment. Undetectable ctDNA was associated consistently with a numerically higher $\mathrm{pCR}$ rate $(\sim 50 \%)$ across all time points compared to detectable ctDNA, although this was not statistically significant (Table 4).

A timeline of ctDNA for all patients with evaluable ctDNA is provided in Figure 3. Among the 16 resected patients with post-chemoradiation ctDNA draws, only 1 of the 12 patients with undetectable ctDNA (8\%) developed disease recurrence, compared to 3 of the 4 patients with detectable ctDNA $(75 \%)(p=0.004)$. Among the 14 resected patients with postoperative ctDNA draws, none of the 9 patients with undetectable ctDNA $(0 \%)$ developed recurrence versus 2 of 5 patients $(40 \%)$ with detectable ctDNA $(p=0.045)$ after surgery.

\section{Tumor Immune Microenvironment}

Only 9 of 25 patients had enough residual pre-treatment baseline tumor after tissue genomic analysis to perform additional tumor microenvironment evaluation. Of the 20 patients who underwent resection, 12 patients had postoperative tissue available for evaluation. One patient with minimal 
residual disease at time of surgery did not have tissue for postoperative tissue evaluation. Supplemental Appendix 6 summarizes the IHC for pre-planned tumor immune microenvironment profiling for the pretreatment and post-treatment specimens. Of these, only 4 patients had paired pre-treatment and posttreatment tissue available for comparison (Supplemental Appendix 7).

\section{Pre- and Post-treatment Molecular Profiles}

Twenty pre-treatment and 13 post-treatment samples were analyzed by SNaPshot molecular profiling, with paired pre- and post-treatment analysis available for 11 patients (Supplemental Appendix 8). Review of variants in pre- and post-treatment samples revealed 3 patients with evidence of potential treatmentinduced subclonal selection and/or tumor evolution (Figure 4). The pre-treatment sample for Patient 25 with a cancer of the gastric antrum demonstrated a KRAS G13D mutation, and this patient was known to have microsatellite instability as a consequence of MLH1/PMS2 methylation-based gene repression, consistent with MSI-high gastric cancer. Post-treatment molecular analysis revealed 8 additional MSI subtype-related mutations, consistent with a hypermutable state (Figure 4A).

The pre-treatment sample for Patient 24 with GE junction cancer possessed molecular variants in TP53, RNF43, AKT1 and SMARCA4. Post-treatment analysis (tumor purity 60\%) detected new PIK3CA (3 distinct mutations), MAP3K1, MYCN and FGFR2 mutations but did not detect the TP53 or RNF43 variants, suggestive of loss of subclone(s) possessing TP53 and RNF alterations (Figure 4B). Numerous PIK3CA mutations were detected in the post-treatment sample, which could reflect: 1) single clone with bi-allelic $\mathrm{H} 1047$ mutations, at least one allele of which co-occurs with a C420R mutation, or 2) multiple clones with different PIK3CA mutation permutations. Of note, the epigenetic disruption from SMARCA4 mutations has been previously reported to result in gastric tumor subclonal subtype plasticity ${ }^{29}$, which may be reflected in the numerous molecular variants evident on post-treatment sequencing.

Patient 19 with a GE junction adenocarcinoma demonstrated no variants on pre-treatment sample analysis (tumor purity 70\%), while post-treatment analysis indicated 4 molecular variants (TP53, KRAS amplification, PIK3CA and FBX7W). Analysis of TCGA gastric tumor data (Supplemental Appendix 9) revealed that none of the PIK3CA mutant TCGA gastric tumors possessed KRAS amplifications, and vice versa. Additionally, KRAS amplified and TP53 mutations clustered in CIN subtype tumors, PIK3CA and FBXW7 mutations were associated with MSI tumors, and co-mutational statuses were even more specific for these two subtypes. Assessing Patient 19's tumor evolution using this information suggests two models of tumor evolution (Figure 4C): 1) typical "accumulation" of mutations, and 2) establishment and outgrowth of 2 putative subclones of different subtypes.

\section{Discussion}

There are currently multiple different treatment paradigms, all supported by randomized controlled trials, which are deemed acceptable as standard of care treatment for locally advanced GE junction and gastric cancers $^{3-9}$. For GE junction cancers, the CROSS study established neoadjuvant chemoradiation with 
concurrent carboplatin/paclitaxel as one acceptable standard of care for treatment of locally advanced distal esophageal and GEJ cancers, yielding improved OS compared to surgery alone ${ }^{9}$. Perioperative chemotherapy alone is another acceptable treatment for GE junction and gastric cancers, having demonstrated improved outcomes compared to surgery alone ${ }^{5}$. More recently, the FLOT-4 study established the superiority of perioperative FLOT (5-FU, docetaxel, and oxaliplatin) to perioperative ECF (epirubicin, cisplatin, and 5-FU) ${ }^{4}$. However, perioperative approaches are plagued by low rates of receipt of post-operative therapy, potentially limiting efficacy ${ }^{3-6}$. Despite these promising advancements in the treatment of gastric and GEA cancers, the 5 -year OS is still only in the range of $40-50 \%^{3-6,9}$. The majority of patients with recurrence will subsequently die of metastatic disease, suggesting a need for systemic intensification to reduce recurrence rates and to prolong survival ${ }^{3,5,30}$.

To address current unmet needs, we performed a pilot study of neoadjuvant FOLFIRINOX followed by consolidative chemoradiation with carboplatin/paclitaxel. We determined that completion of this total neoadjuvant regimen was feasible, with an $88 \%$ therapy completion rate, successfully meeting our primary endpoint. Although we noted a grade 4 toxicity rate of $80 \%$, the majority of grade 4 toxicity was attributable to subclinical lymphopenia (76\%) during induction FOLFIRINOX without clinical implications. The remaining grade 4 toxicity was attributable to only 2 patients (8\%) with grade 4 febrile neutropenia. Overall, the toxicity profile is consistent with that reported in the literature for the various treatment components $^{31}$. While FOLFIRINOX has been shown to be one of most effective regimens in the treatment of pancreatic cancer ${ }^{13,14}$, there has been little published data regarding its efficacy for gastroesophageal and gastric cancers. In 2017, a pooled analysis of two ongoing Phase II trials of FOLFIRINOX in the first line metastatic setting for GE junction cancers demonstrated an overall response rate of $62.5 \%{ }^{15}$. More recently, in a phase II trial from Washington University (NCT 01928290), among ERBB2-negative patients treated with FOLFIRINOX alone, the ORR was $61 \%$, with a median overall survival of 15.5 months. Among ERBB2-positive patients treated with FOLFIRINOX and trastuzumab, the ORR was $85 \%$, with a median OS 19.6 months, ${ }^{16}$ which offers promising results compared to historical controls ${ }^{24}$. Additionally, a multiinstitutional phase II trial of 36 patients with locally advanced adenocarcinoma evaluated pharmacogenomically dosed perioperative FOLFIRINOX and reported RO resection rate of $92 \%$ and a pCR rate of $23 \%{ }^{17}$. These studies support the safety and early clinical activity of FOLFIRINOX in gastroesophageal adenocarcinomas. To obviate concerns about tolerability and compliance with adjuvant therapy, we opted to pursue a total neoadjuvant approach. Our study mimics the movement in currently ongoing randomized trials in this space, including the CRITICS-II ${ }^{10}$ and TOPGEAR ${ }^{11}$ studies, which frontload chemotherapy and chemoradiation prior to surgical resection.

Moreover, in our small series, the pCR rates (28\% in the ITT group and $35 \%$ in the resected group) compare favorably to those achieved with other currently accepted neoadjuvant strategies. In the FLOT 4 study, which evaluated the efficacy of perioperative 5-FU, oxaliplatin and docetaxel, the rate of pCR reported after 4 cycles of neoadjuvant FLOT was $16 \%{ }^{4}$. More recently, perioperative FOLFIRINOX yielded a pCR rate of $23 \%{ }^{17}$. As shown in the POET study, the addition of neoadjuvant chemoradiation compared 
to neoadjuvant chemotherapy alone improves rates of $\mathrm{pCR}^{33}$. Therefore, it is perhaps not surprising that among studies which include neoadjuvant radiation, the $\mathrm{pCR}$ rate was $23 \%{ }^{9}$ in the CROSS trial and $26 \%$ for RTOG $9904^{34}$, respectively. Numerous studies have suggested a correlation between PCR and histologic treatment response and long-term disease outcomes ${ }^{34,35}$. In addition to pCR, nodal clearance has also been suggested to be prognostic in the setting of perioperative therapy ${ }^{36}$. In our current study, although $80 \%$ of patients at baseline had clinically involved lymph nodes, only $30 \%$ were found with pathologically involved lymph nodes, suggesting the impact of treatment on nodal clearance. Additionally, all patients achieved an R0 resection (100\%), which compare s favorably to R0 resection rates seen with perioperative FLOT or ECX (R0 rates of $85 \%$ vs $74 \%$, respectively) ${ }^{4}$. Longer term followup of the patients enrolled in this current study will be necessary to determine the impact of dose intensification of FOLFIRINOX on long-term distant disease control.

Although our current study had limited events, ctDNA appears as a promising predictor of disease recurrence. Despite the small number of patients and events, we found that detectable ctDNA after chemoradiation $(p=0.004)$ and postoperatively $(p=0.045)$ were associated with disease recurrence. Analysis of ctDNA after completion of definitive therapy has been shown to be promising in detection of minimal residual disease in several disease sites to date ${ }^{37-42}$. Recent retrospective data from MDACC has emerged that suggest that post-chemoradiation ctDNA may identify patients with localized esophageal cancers at increased risk of recurrence and death and may potentially help in the determination of which patients are most likely to benefit from surgery. Our study, however, represents to our knowledge the first prospective series to demonstrate the prognostic value of ctDNA in patients with locally advanced esophagogastric cancer treated with total neoadjuvant therapy.

Limited molecular data are available to assess molecular factors that contribute to tumor evolution and subclonal selection during CRT for gastric and GEA tumors ${ }^{29}$. While analysis of large tumor bank sequencing data for untreated GEA and gastric tumors has revealed the presence of diverse tumor subtypes within a given anatomic location ${ }^{43}$, it is unclear how these subtypes or their molecular hallmarks might contribute to treatment resistance or to the evolution of tumors over the course of treatment. Our exploratory molecular analysis of pre- and post-treatment tumor samples indicates numerous putative or potential models of tumor evolution and suggests heterogeneous responses of subclonal populations to neoadjuvant CRT. These models are suggested by our data, but limitations of our technique prevent conclusive establishment of tumor evolution patterns in this study. Our analysis is limited by reduced sensitivity due to variable sample purity and the acquisition of only a single analyzed sample for each time point, and underlying tumor heterogeneity cannot be excluded. However, our data provides support warranting future single-cell and spatial transcriptomic analyses from pre- and posttreatment samples to characterize patterns of treatment adaptation and resistance to inform individualized therapeutic approaches.

Lastly, given the emerging awareness of the importance of the tumor immune microenvironment, we performed an exploratory analysis looking to profile and compare pre-treatment baseline and post- 
treatment tumor immune microenvironments by examining PD-L1, PD-1, CD8, CD8 TIL, CD3 and FOXP3 expression. In our very limited series of paired specimens, we did not find any particular trends in tumor infiltration of immune cells, with some patients demonstrating similar $(n=2)$, increased $(n=1)$, and decreased $(n=1)$ extent of tumor infiltrates. Future, larger patient cohorts will hopefully further elucidate and explore the prognostic implications of the tumor immune microenvironment with potential therapeutic implications.

In conclusion, in this prospective single-institution pilot study, neoadjuvant FOLFIRINOX followed by CRT is feasible and safe, and encouraging preliminary antitumor activity is highlighted by the high pCR rate and nodal clearance at surgery. A follow up study is currently planned. Lastly, although our current study is limited by events, post-CRT and postoperative ctDNA appears to be a promising biomarker to predict disease recurrence. Future studies investigating the role of ctDNA and the potential for tumor immune biomarkers in the management of gastric and GEA cancers are warranted to corroborate our findings.

\section{Declarations}

Conflicts of Interest: JYW: Research funding: Genentech; Honorarium: Physician's Educational Resource; Lynx Group; JWC: None; CEE: None; MMK: Consultant: H3 Biomedicine (consultant); AstraZeneca (consultant); (Novartis) Research, not related to the current study; SJK: has served a consultant/advisory role for Bristol Myers Squibb, Merck, Eli Lilly, Astellas, Pieris, and Foundation Medicine, Inc. SJK reports stock/equity in Turning Point Therapeutics; JNA: None; FKK: UpToDate (honorarium); OncLive (honorarium); AstraZeneca (advisory board); Merck (research funding), not related to current study; ARP: Consulting/Advisory Board: Eli Lilly, Natera, Checkmate, Pfizer; DSMC: Genentech; Research to institution: Guardant, Natera, Array, Eli Lilly, BMS, Tesaro, Novartis, Macrogenics, PMV Pharmaceuticals, Takeda, Plexxicon; ER: Consultant for Mitobridge Inc., Asahi Kasei Pharmaceuticals, DRG Consulting, Napo Pharmaceuticals, American Imaging Management, Immuneering Corporation, Prime Oncology; additionally, he has served on recent advisory boards for Heron Pharmaceuticals, Vector Oncology; and has served as a member on data safety monitoring boards for Oragenics, Inc, Galera Pharmaceuticals, and Enzychem Lifesciences Pharmaceutical Company; LCD: None; DPR: Equity: MPM Capital, Acworth Pharmaceuticals, Thrive Earlier Detection; Advisory: MPM Capital, Oncorus, Gritstone Oncology, Maverick Therapeutics, 28/7 Therapeutics; Publishing: Johns Hopkins University Press, Uptodate, McGraw Hill; Legal Consulting: Boeringer-Ingelheim, Iteos; RBC: Consultant/advisory board member: Amgen, Array Biopharma, Astex Pharmaceuticals, Avidity Biosciences, BMS, C4 Therapeutics, Chugai, Elicio, Fog Pharma, Kinnate Biopharma, Genentech, LOXO, Merrimack, N-of-one, Novartis, nRichDx, Revolution Medicines, Roche, Roivant, Shionogi, Shire, Spectrum Pharmaceuticals, Symphogen, Taiho, and Warp Drive Bio; Equity in Avidity Biosciences, C4 Therapeutics, Kinnate Biopharma, nRichDx, and Revolution Medicines; Research funding from Asana, AstraZeneca, and Sanofi; EVS: None; IF: None; HAS: None; MK: None; ML: Bristol Myers Squibb (consultant); CRM: None; RSH: Honoraria: Novartis, Merck Healthcare $\mathrm{KGaA}$, Pfizer, Roche, Apollomics, Tarveda, Boehringer Ingelheim; Research funding to institution, not to self: Novartis, Genentech Roche, Corvus, Incyte, Exelixis, Abbvie, Daichii Sankyo, Agios, Mirati, Turning Point, Lilly. CAA: None; CM: None; BC: None; BYY: None; JTM: None; TSH: Consulting: Novocure, Merck, 
Synthetic biologics; Research funding to institution: Ipsen, Tesaro, Astra-Zeneca,BMS, IntraOp, Taiho, Puma.

Funding: This study was funded by NIH Proton Beam NCI/Federal Share Program grant C06 CA059267, and in part by the Cancer Clinical Investigator Team Leadership Award awarded by the National Cancer Institute though a supplement to P30CA006516 (TSH)

ClinicalTrials.gov Identifier: NCT03279237

Statistical Analysis: Beow Y. Yeap, ScD and Christine A. Ulysse, SM

Conflicts of Interest: None

Authors Contribution: Dr. Wo and Dr. Hong had full access to all the data in the study and take responsibility for the integrity of the data and the accuracy of the data analysis.

Study concept and design: Ryan, Hong, Clark, Mullen.

Acquisition, analysis or interpretation of data: Wo, Hong, Van Seventer, Eyler, Mino-Kenudsen

Drafting of Manuscript: Wo, Hong, Van Seventer, Eyler, Mino-Kenudsen, Mullen.

Critical revision of the manuscript for important intellectual content: All authors.

Statistical analysis: Yeap, Ulysse.

Funding: Hong.

Administrative, technical, or material support: Wo, Ryan, Klempner, Hong, Corcoran, Parikh.

Study Supervision: Hong, Wo, Yeap, Mullen.

\section{References}

1. National Cancer Institute (2019). Cancer stat facts: gastric cancer. Retrieved from: https://seer.cancer.gov/statfacts/html/stomach.html.

2. National Cancer Institute (2019). Cancer stat facts: esophageal cancer. Retrieved from: https://seer.cancer.gov/statfacts/html/esoph.html.

3. Smalley, S.R., et al. Updated analysis of SWOG-directed intergroup study 0116: a phase III trial of adjuvant radiochemotherapy versus observation after curative gastric cancer resection. J Clin Oncol. 30, 2327-2333 (2012).

4. Al-Batran, S.E. et al. Perioperative chemotherapy with fluorouracil plus leukovorin, oxaliplatin, and docetaxel versus fluorouracil or capecitabine plus cisplatin and epirubicin for locally advanced, 
resectable gastric or gastro-oesophageal junction adenocarcinoma (FLOT4): a randomised, phase 2/3 trial. Lancet 393, 1948-1957 (2019).

5. Cunningham, D. et al. Perioperative chemotherapy versus surgery alone for resectable gastroesophageal cancer. N Engl J Med. 355, 11-20 (2006).

6. Cats, A. et al. Chemotherapy versus chemoradiotherapy after surgery and preoperative chemotherapy for resectable gastric cancer (CRITICS): an international, open-label, randomised phase 3 trial. Lancet Oncol. 19, 616-628 (2018).

7. Noh, S.H. et al. Adjuvant capecitabine plus oxaliplatin for gastric cancer after D2 gastrectomy (CLASSIC): 5- year follow-up of an open-label, randomised phase 3 trial. Lancet Oncol. 15, 13891396 (2014).

8. Lee, J. et al. Phase III trial comparing capecitabine plus cisplatin versus capecitabine plus cisplatin with concurrent capecitabine radiotherapy in completely resected gastric cancer with D2 lymph node dissection: the ARTIST trial. J Clin Oncol. 30, 268-73 (2012).

9. van Hagen, P. et al. Preoperative chemoradiotherapy for esophageal or junctional cancer. N Engl J Med. 366, 2074-2084 (2012).

10. Slagter, A.E. et al. CRITICS-II: a multicentre randomised phase II trial of neoadjuvant chemotherapy followed by surgery versus neoadjuvant chemoradiotherapy followed by surgery in resectable gastric cancer. BMC Cancer. 18, 877-88 (2018).

11. Leong , T. et al. Preoperative Chemoradiation for Resectable Gastric Cancer: Interim Results from an International, Intergroup Trial of AGITG, TROG, EORTC, and CCTG. Ann Surg Oncol. 24, 2252-2258 (2017).

12. Mizrak Kaya, D. et al. Efficacy of Three-Drug Induction Chemotherapy Followed by Preoperative Chemoradiation in Patients with Localized Gastric Adenocarcinoma. Oncology. 98, 542-548 (2020).

13. Conroy, T. et al. FOLFIRINOX versus gemcitabine for metastatic pancreatic cancer. N Eng/ J Med. 364, 1817-1825 (2011).

14. Conroy, T. et al. FOLFIRINOX or gemcitabine as adjuvant therapy for pancreatic cancer. N Engl J Med. 379, 2395-2406 (2018).

15. Lockhart, A.C. et al. FOLFIRINOX as first-line therapy in patients with metastatic gastroesophageal cancers (GEC). J Clin Oncol. 33, 177 (2015).

16. Park, H. et al. FOLFIRINOX for the treatment of advanced gastroesophageal cancers: a phase 2 nonrandomized clinical trial. JAMA Oncol. 6, 1231-40 (2020).

17. Catenacci, D.V.T. et al. Evaluation of the association of perioperative UGT1A1 Genotype-dosed gFOLFIRINOX with margin negative resection rates and pathologic response grades among patients with locally advanced gastroesophageal adenocarcinoma: a phase 2 clinical trial. JAMA Open Net. 3, e1921290 (2020).

18. Lowy, A.M. et al. Response to neoadjuvant chemotherapy best predicts survival aftercurative resection of gastric cancer. Annals of Surgery. 229, 303-308 (1999). 
19. Huynh, T.G. et al. Programmed Cell Death Ligand 1 Expression in Resected Lung Adenocarcinoma: Association with Immune Microenvironment. J Thorac Oncol. 11, 1869-1878 (2016).

20. Zheng, Z. et al. Anchored multiplex PCR for targeted next-generation sequencing. Nat Med. 20, 147984 (2014).

21. Cibulskis, K. et al. Sensitive detection of somatic point mutations in impure and heterogeneous cancer samples. Nat Biotechnol. 31, 213-219 (2013).

22. Wilm, A. et al. LoFreq: a sequence-quality aware, ultra-sensitive variant caller for uncovering cellpopulation heterogeneity from high-throughput sequencing datasets. Nucleic Acids Res. 40, 1118911201 (2012).

23. Van der Auwera, G.A, et al. From FastQ data to high confidence variant calls: the Genome Analysis Toolkit best practices pipeline. Curr Protoc Bioinformatics. 43, 11.10.1-11.10.33, (2013).

24. Cancer Genome Atlas Research Network. Comprehensive molecular characterization of gastric adenocarcinoma. Nature 513: 202-209 (2014).

25. Cerami, E. et al. The cBio Cancer Genomics Portal: An Open Platform for Exploring Multidimensional Cancer Genomics Data. Cancer Discov. 2, 401-4 (2012).

26. Goldman, M.J. et al. Visualizing and interpreting cancer genomics data via the Xena platform. Nat Biotechnol. 38, 675-678 (2020).

27. Murugaesu, N. et al. Tracking the genomic evolution of esophageal adenocarcinoma through neoadjuvant chemotherapy. Cancer Discov. 5, 821-831 (2018).

28. Oppedijk, V. et al. Patterns of recurrence after surgery alone versus preoperative chemoradiotherapy and surgery in the CROSS trials. J Clin Oncol. 32, 385-391 (2014).

29. Davuluri, R. et al. Lymphocyte Nadir and Esophageal Cancer Survival Outcomes After Chemoradiation Therapy. IJROBP. 99, 128-135 (2017).

30. Bang YJ, et al. Trastuzumab in combination with chemotherapy versus chemotherapy alone for treatment of Her2-positive advanced gastric or gastroesophageal junction cancer (ToGA): a phase III, open label, randomized controlled trial. Lancet 376, 687-697 (2010).

31. Stahl, M et al. Phase III Comparison of Preoperative Chemotherapy compared with Chemoradiotherapy in Patients with Locally advanced Adenocarcinoma of the Esophagogastric Junction. J Clin Oncol. 27, 851-856 (2009).

32. Ajani, J.A. et al. Phase II trial of preoperative chemoradiation in patients with localized gastric adenocarcinoma (RTOG 9904): quality of combined modality therapy and pathologic response. $J$ Clin Oncol. 24, 3953-3958 (2006).

33. Li, Z. et al. Correlation of pathological complete response with survival after neoadjuvant chemotherapy in gastric or gastroesophageal junction cancer treated with radical surgery: A metaanalysis. PLoS One. 13, e0189294 (2018).

34. Smyth, E.C. et al. Effect of pathologic tumor response and nodal status on survival in the Medical Research Council Adjuvant Gastric Infusional Chemothearpy Trial. J Clin Oncol.34, 2721-2727 
(2006).

35. Garcia-Murillas, I. et al. Mutation tracking in circulating tumor DNA predicts relapse in early breast cancer. Sci Trans/ Med. 7:302ra133 (2015).

36. Tie, J. et al. Circulating tumor DNA analysis as markers of recurrence risk and benefit of adjuvant therapy for Stage III colon cancer. JAMA Oncol. 5, 1710-1717 (2019).

37. Chaudhuri, A.A, et al. Early Detection of Molecular Residual Disease in Localized Lung Cancer by Circulating Tumor DNA Profiling. Cancer Discov. 7:1394-1403 (2017).

38. Azad, T.D. et al. Circulating tumor DNA analysis for detection of minimal residual disease after chemoradiotherapy for localized esophageal cancer. 58, 494-505 (2020).

39. Yang, J. et al. Deep sequencing of circulating tumor DNA detects molecular residual disease and predicts recurrence in gastric Cell Death Dis. 11: 346, (2020).

40. Leal, A. et al. White blood cell and cell-free DNA analyses for detection of residual disease in gastric cancer. Nat Commun. 11:525 (2020).

41. Liu, Y. et al. Comparative Molecular Analysis of Gastrointestinal Adenocarcinomas. Cancer Cel/ 33, 721-35 (2018).

\section{Tables}

Table 1. Patient and Tumor Characteristics. 
Characteristics

Age, median (range)

Gender

Male

Female

ECOG PS

0

1

Primary Tumor Location

GE Junction

Gastric

Cardia

Fundus

Body

Antrum

Diffuse

Clinical TN Stage

T3N0

T4N0

T1-4,N1-3

Nodal M1 Disease

Baseline CEA, median (range)

Baseline CA 19-9, median (range) $16(<1-8004)$

Histologic Subtype

Diffuse

Signet Ring

Adenocarcinoma, NOS

Her2 Status

Positive

Negative

Not Available/Equivocal
Number (\%)

$60(30-76)$

17 (68\%)

$8(32 \%)$

$16(64 \%)$

9 (36\%)

$15(60 \%)$

$10(40 \%)$

1

1

3

4

1

3 (12\%)

$2(8 \%)$

$20(80 \%)$

7 (28\%)

$2.6(0.4-251.7)$

1 (4\%)

5 (20\%)

19 (76\%)

3 (12\%)

17 (68\%)

5 (20\%)

Table 2. Surgical Outcomes ( $\mathrm{n}=20$ resected). 
Surgery type

Thoracoabdominal esophagectomy

$5(25)$

Minimally invasive esophagectomy

$4(20)$

Ivor-Lewis esophagectomy

$5(25)$

Total gastrectomy

2 (10)

Distal gastrectomy

$4(20)$

Margin status

R0

$20(100)$

R1

$0(0)$

Pathologic tumor size $(\mathrm{cm})$, median (range)

$0.25(0.0-7.7)$

Pathologic tumor size $(\mathrm{cm})$ excluding pCR, median (range) $2.3(<0.1-7.7)$

Pathologic stage

TONO (resected)

7 (35)

T1 N0

$3(15)$

T2N0

$3(15)$

T3N0

$1(5)$

$\mathrm{T} 1-3, \mathrm{~N} 1-3$

$6(30)$

Lymph nodes involved, median (range)

$0(0-7)$

Lymph nodes dissected, median (range)

$22(11-43)$

Number of lymph nodes dissected

$$
\begin{aligned}
& <15 \\
& \geq 15
\end{aligned}
$$

$15(75)$

Table 3. Acute toxicity related to chemotherapy and chemoradiation. 


\begin{tabular}{|c|c|c|c|}
\hline CTCAE 4.0 category & Grade 3 & Grade 4 & Grade 3-5 \\
\hline Lymphopenia/Leukopenia/Neutropenia & 2 & 19 & 21 \\
\hline Febrile neutropenia & 0 & 2 & 2 \\
\hline Thrombocytopenia & 4 & 0 & 4 \\
\hline Anemia & 3 & 0 & 3 \\
\hline Hypokalemia/Hyponatremia & 5 & 0 & 5 \\
\hline Dehydration & 3 & 0 & 3 \\
\hline Weight loss & 3 & 0 & 3 \\
\hline Anorexia & 2 & 0 & 2 \\
\hline Nausea/Vomiting & 4 & 0 & 4 \\
\hline Diarrhea & 4 & 0 & 4 \\
\hline Dysphagia & 1 & 0 & 1 \\
\hline Mucositis oral & 1 & 0 & 1 \\
\hline Gastritis & 1 & 0 & 1 \\
\hline Myochosis & 1 & 0 & 1 \\
\hline Infection & 2 & 0 & 2 \\
\hline Hypotension & 2 & 0 & 2 \\
\hline Fatigue & 1 & 0 & 1 \\
\hline ALT elevated & 1 & 0 & 1 \\
\hline Acute kidney injury & 1 & 0 & 1 \\
\hline Atrial fibrillation & 1 & 0 & 1 \\
\hline Aspiration & 1 & 0 & 1 \\
\hline Worst grade overall & $(16 \%)$ & (80\%) & (96\%) \\
\hline
\end{tabular}

Table 4. Correlation of ctDNA to pCR and disease recurrence.

Timepoint Undetectable ctDNA Detectable ctDNA P-value

$\begin{array}{lccc}\text { C1D1 } & 50 \%(2 / 4) & 29 \%(5 / 17) & 0.574 \\ \text { C1D8 } & 50 \%(6 / 12) & 11 \%(1 / 9) & 0.159 \\ \text { Post-CRT* } & 46 \%(6 / 13) & 25 \%(1 / 4) & 0.596 \\ & \text { Recurrence (crude rate ) of resected patients } & * * \\ \text { Post-CRT* } & 8 \%(1 / 12) & 75 \%(3 / 4) & 0.004 \\ \text { Post-Operative } & 0 \%(0 / 9) & 40 \%(2 / 5) & 0.045\end{array}$

* One patient completed chemoradiation but was not resected due to intraoperative metastases. **P-value based on cumulative incidence of distant metastases. 
Abbreviations: ctDNA: circulating tumor DNA; pCR: pathologic complete response; C1D1: cycle 1 day 1; CRT: chemoradiation

\section{Figures}

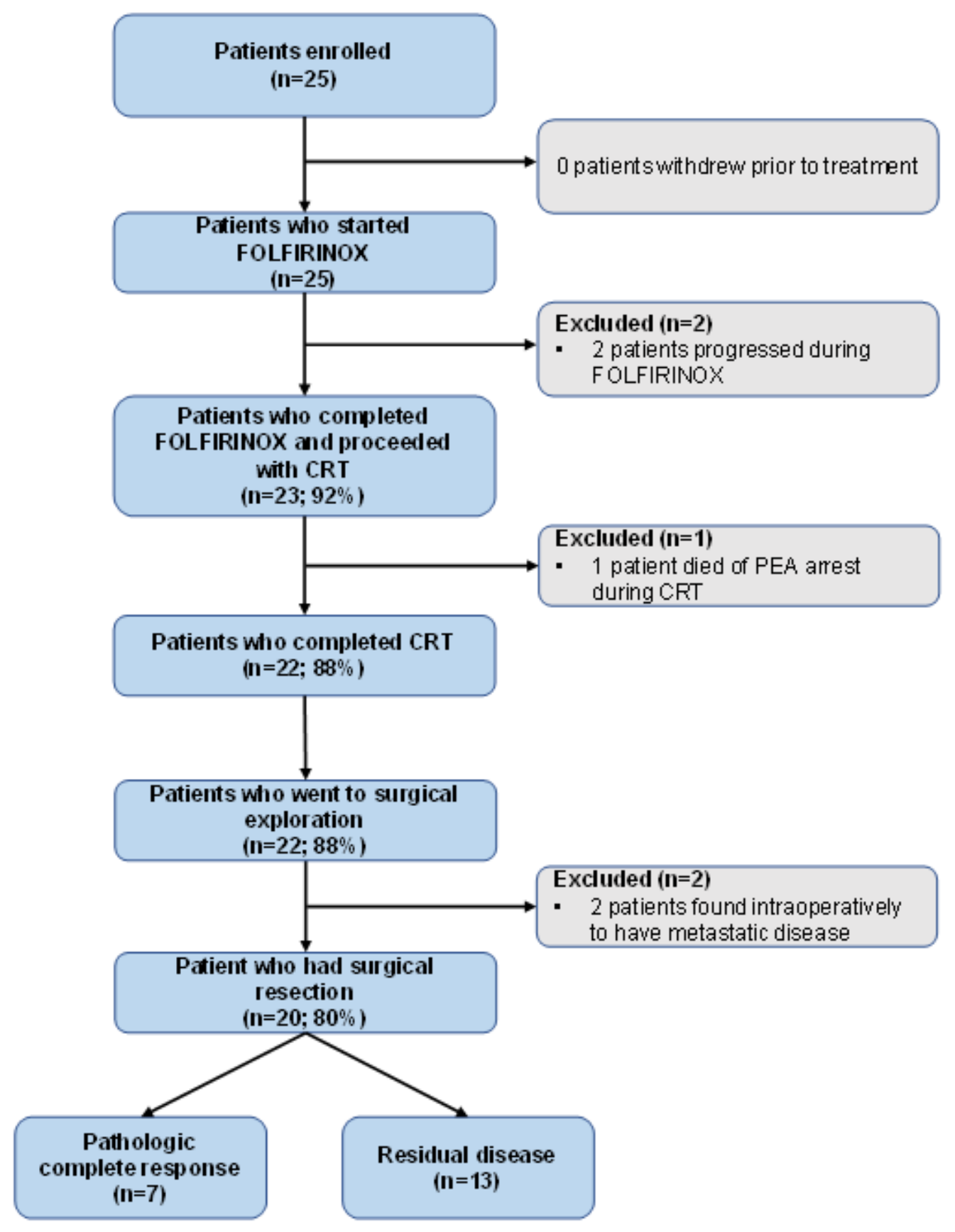

Figure 1

CONSORT Diagram. 
$2 a$.

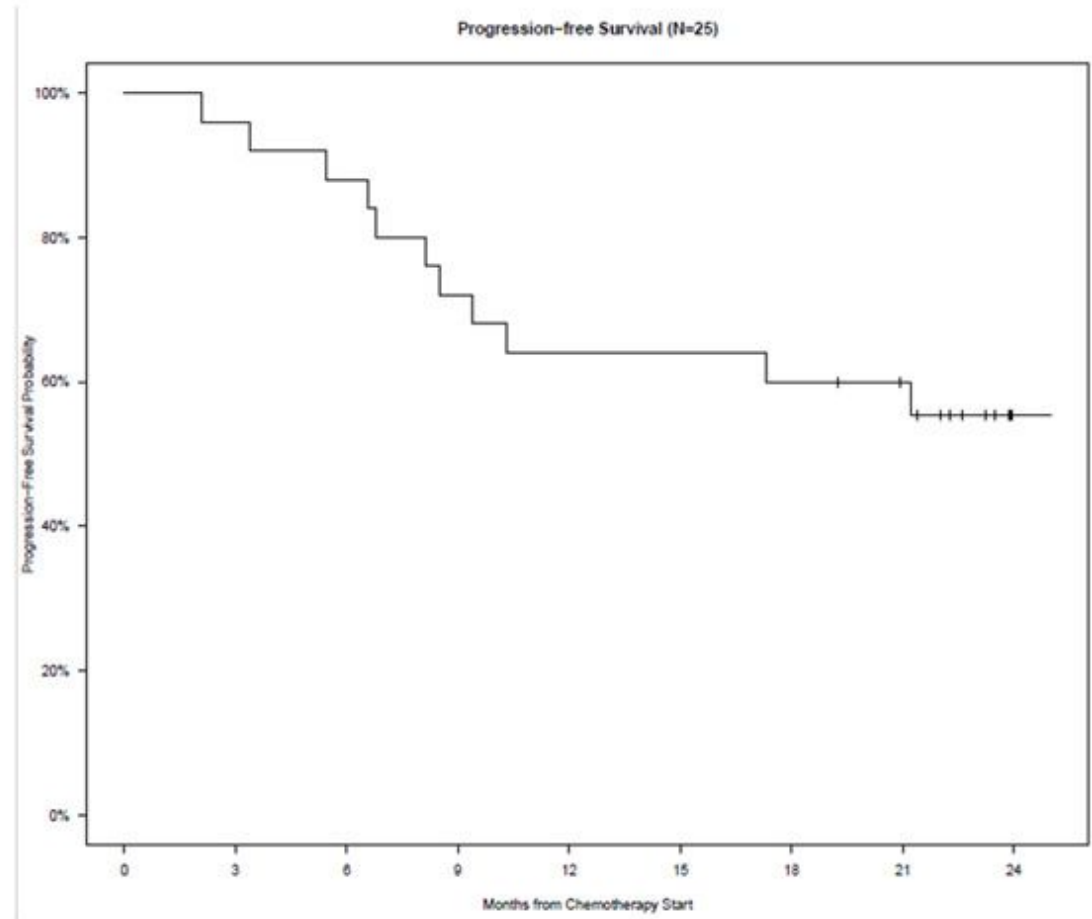

$2 \mathrm{~b}$.

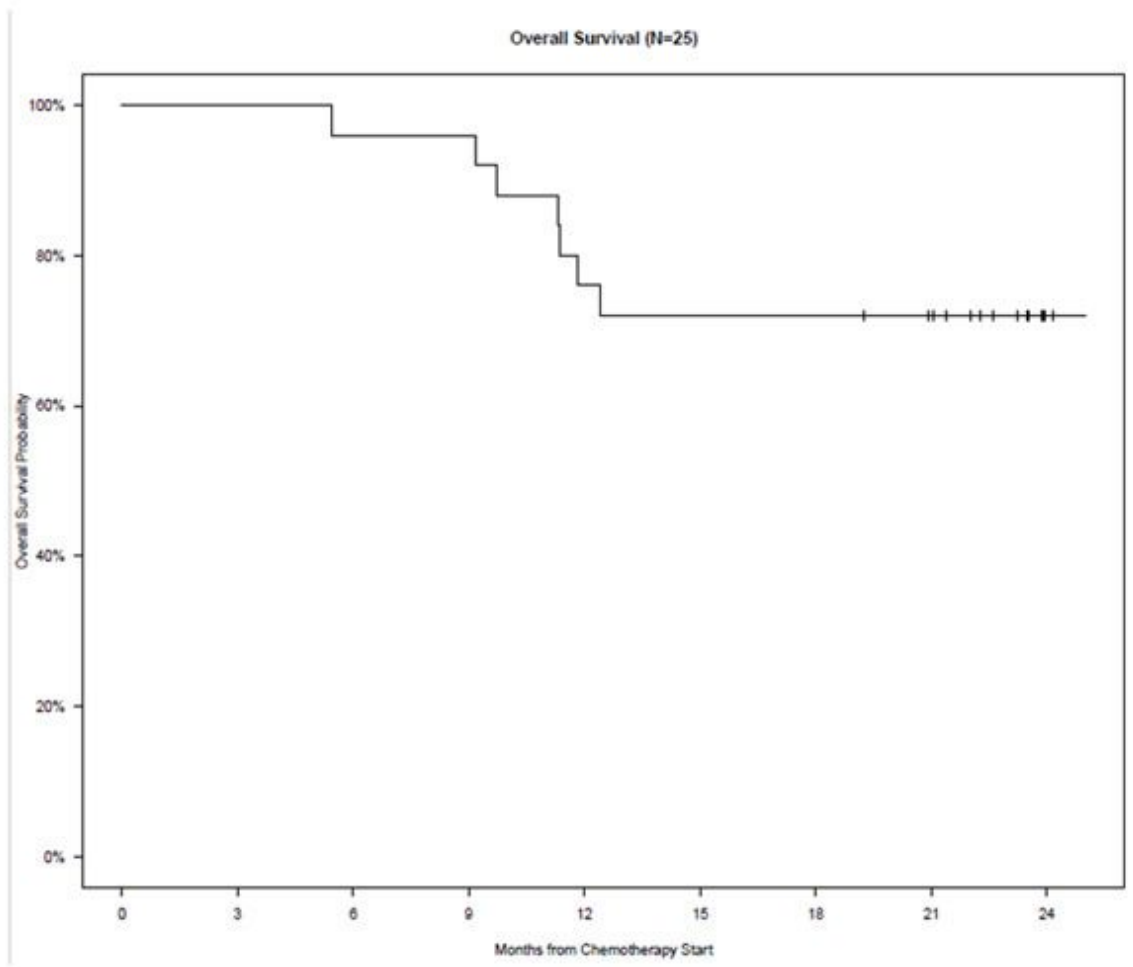

Figure 2

Kaplan-Meier curves for (a) PFS and (b) OS 


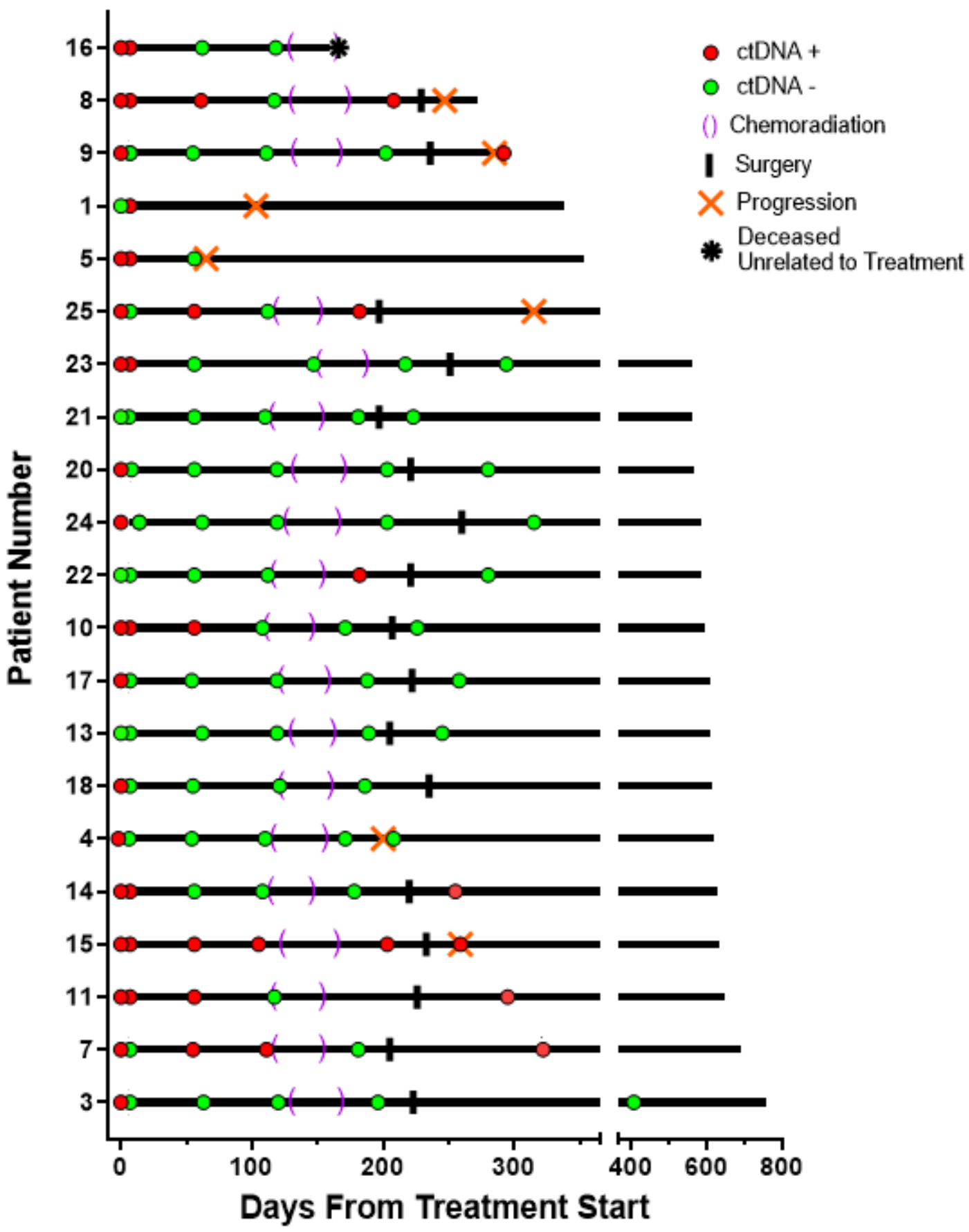

Figure 3

Time of ctDNA for all evaluable patients $(n=21)$ correlated to treatment and disease status. 
$4 a$

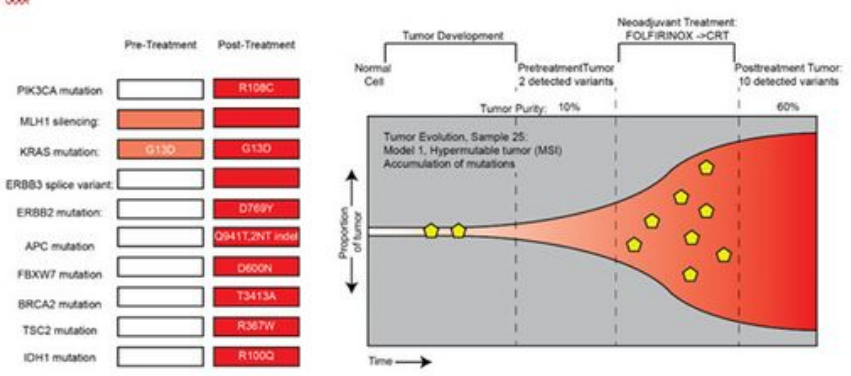

4b.

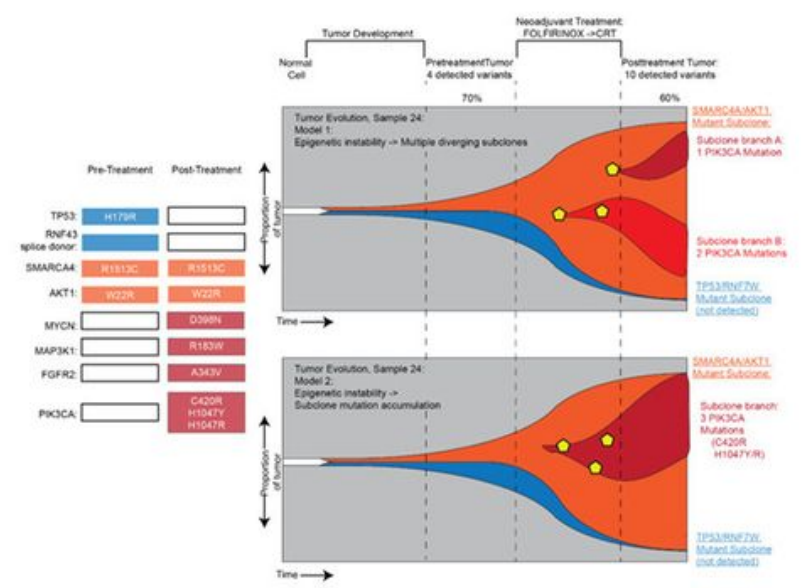

4c.

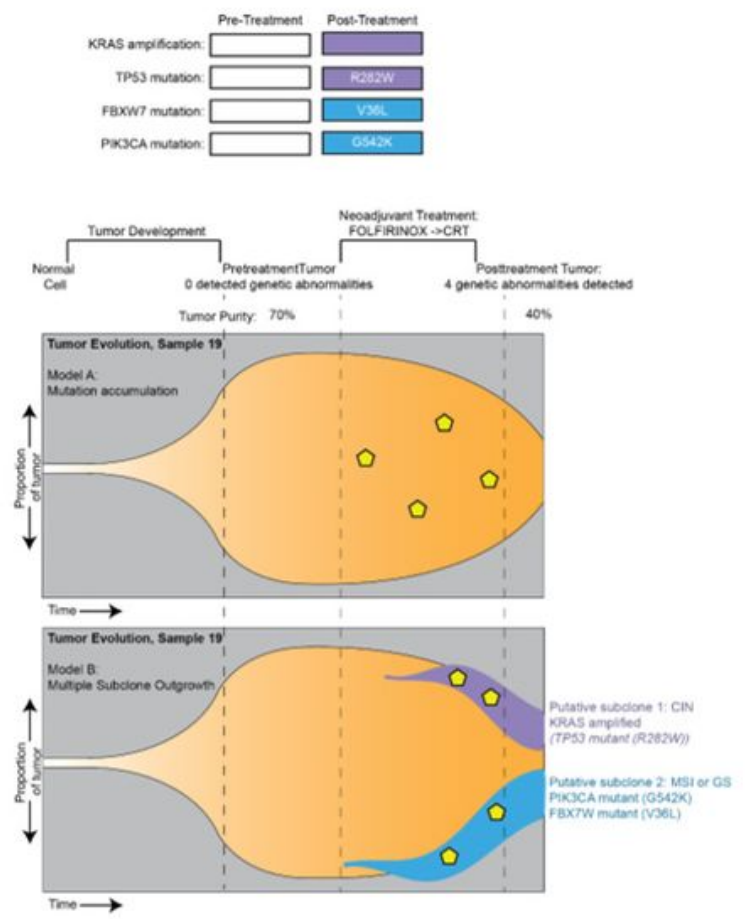

Figure 4

Treatment-induced tumor evolution

\section{Supplementary Files}

This is a list of supplementary files associated with this preprint. Click to download. 
- 17311Protocol.pdf

- Supplementallnformation.docx 